\title{
Inflationary Cosmology in RS-I
}

\author{
Michele Ferraz Figueiró \\ Instituto de Física, \\ Universidade de São Paulo \\ Caixa Postal 66318, 05315-970, São Paulo-SP, Brazil
}

(Received on 17 October, 2005)

\begin{abstract}
In this work, I intend to show a possible candidate of inflaton potential $V(\phi)$ in a scenario of a brane world defined by a pair of branes (RS-I).
\end{abstract}

The Inflationary Cosmology describes a phase in which our Universe evolves through accelerated expansion in a short time period at high energy scales. During this phase, our Universe is dominated by a potential $V(\phi)$ generated by a homogeneous scalar field $\phi(t)$ called inflaton. This potential must obey slow-roll conditions $\{\varepsilon,|\eta| \ll 1\}$ where $\varepsilon$ and $\eta$ are the slow-roll parameters. These parameters are given by [1]

$$
\varepsilon(\phi)=\frac{M_{P L}^{2}}{2}\left(\frac{V^{\prime}}{V}\right)^{2}
$$

and

$$
\eta(\phi)=M_{P L}^{2} \frac{V^{\prime \prime}}{V}
$$

We can calculate the spectral index $n(\phi)$ and its derivate for this potential $V(\phi)$

$$
n-1=-6 \varepsilon+2 \eta
$$

and

$$
\frac{d n}{d \ln k}=-16 \varepsilon \eta+24 \varepsilon^{2}+2 \zeta
$$

where

$$
\zeta=M_{P L}^{4} \frac{V^{\prime} V^{\prime \prime \prime}}{V^{2}}
$$

The amount of inflation that occurs is described by the number of e-foldings $N$, is given by

$$
N \equiv \ln \frac{a\left(t_{\text {end }}\right)}{a(t)} \equiv \int_{t}^{t_{\text {end }}} H d t \approx \frac{1}{M_{P L}^{2}} \int_{\phi_{\text {end }}}^{\phi} \frac{V}{V^{\prime}} d \phi,
$$

where $\phi_{\text {end }}$ is defined by $\varepsilon\left(\phi_{\text {end }}\right)=1$ if inflation ends through violation of the slow-roll conditions.

The Brane Cosmology describes cosmological models with extra dimensions. A lot of interest in brane cosmology arose with a publication of two papers by Randall and Sundrum in the 90s. They propose a new higher-dimensional mechanism for solving the hierarchy problem building two models, $R S-I$ [2] and $R S-I I$ [3]. In these two models, they consider that the Standard Model particles and forces, with exception of gravity are confined to a four-dimensional subspace, within the fivedimensional spacetime (bulk), referred to as 3-brane. Many researches have been done around this new cosmology as e.g. [9]. We choose the inflation in branes [4], [6], [7], [8].

In this study, we consider that inflation might arise from the interaction potential between a 3-brane and anti-3-brane which are parallel and widely separated in five-dimensional Anti de Sitter space $\left(A d S_{5}\right)$. The background is identical to that considered in the RS-I model [2].

The potential between the branes is given by [4]

$$
V(\phi) \sim M_{5 D}^{4} \xi\left(\phi / M_{5 D}\right)\left(1-e^{(-|\phi| / m)}\right) .
$$

The figures 1 and 2 show us the behaviours of the potential $V(\phi)$ and of the slow-roll parameters as a function of $\phi$.

The spectral index $n$ and its derivate $d n / d l n k$ can be related to $N$, respectively, as

$$
\frac{1}{2}(n-1)=-\frac{1}{N}
$$

and

$$
\frac{1}{2} \frac{d n}{d \ln k}=-\frac{1}{N^{2}}
$$

Setting $N=70$ (as usually done in inflationary scenarios) leads to

$$
n \approx 0,9714
$$

and

$$
\frac{d n}{d \ln k} \approx-0,0004
$$

in excellent agreement with observational data from WMAP $(0.94 \leq n \leq 1.00$ and $-0.02 \leq d n / d \operatorname{lnk} \leq 0.02,[5])$

\section{Acknowledgement}

This work was supported by CNPq. 


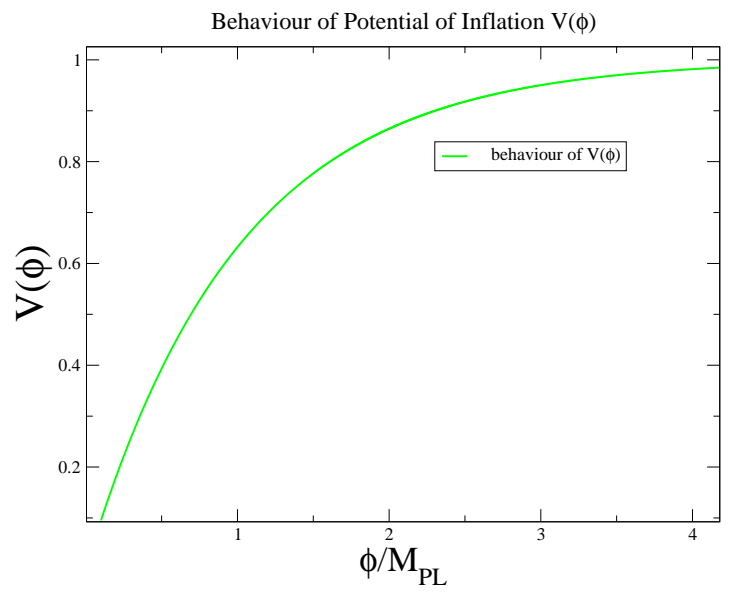

FIG. 1: Behaviour of the potential $V(\phi)$ as a function $\phi$.

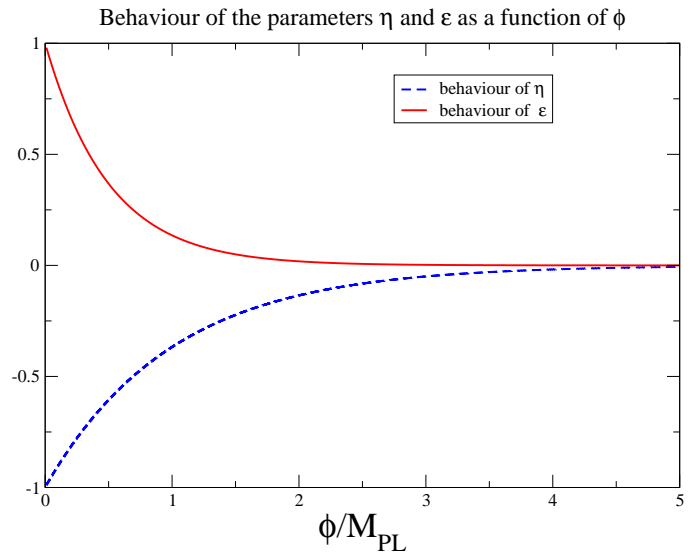

FIG. 2: Behaviour of the slow-roll parameters $\varepsilon$ and $\eta$ as a function of $\phi$.

[1] A. R. Liddle, and D. H. Lyth, Cosmological Inflation and Large-Scale Structure, Cambridge University Press, Cambridge (2000).

[2] L. Randall and R. Sundrum, Phys. Rev. Lett. 83, 3370 (1999).

[3] L. Randall and R. Sundrum, Phys. Rev. Lett. 83, 4690 (1999).

[4] G. Dvali and S.-H Henry Tye, Phys. Lett. B 450, 72 (1999).

[5] H. V. Peiris, et al., Astrophys. J. Suppl. 148, 213 (2003).

[6] S. Kachru, R. Kallosh, and J. Maldacena, JCAP 0310, 013
(2003).

[7] C. Kolda, and D. H. Lyth, D-term Inflation and M-theory, [hep$\mathrm{ph} / 98122343]$

[8] B. Wang, C. Y. Lin, and E. Abdalla, Phys. Rev. D 69, 063507 (2004).

[9] E. Abdalla, A. G. Casali, B. Cuadros-Melgar, Int. J. Theor. Phys. 43, 801 (2004). 